\title{
STEINHAUS-TYPE PROPERTY FOR A BOUNDARY OF A CONVEX BODY
}

\author{
WOJCIECH JABŁOŃSKI
}

\begin{abstract}
We show that if $U \subset \partial A$ is a neighbourhood of a point $x_{0} \in \partial A$ of the boundary of a convex body $A$ then it has the so-called Stainhaus-type property (int $(U+U) \neq \emptyset$ ) if and only if $x_{0}$ is not a point of flatness of the boundary $\partial A$. This implies that additive functions as well as mid-convex functions, bounded above on $U$, are continuous.
\end{abstract}

\section{INTRODUCTION}

Let $X$ be a real topological vector space. For nonempty sets $A, B \subset X$ and for $\alpha \in \mathbb{R}$ we define the Minkowiski's operations

$$
\begin{aligned}
& A \pm B:=\{a \pm b \in X: a \in A, b \in B\}, \\
& \alpha A:=\{\alpha a \in X: a \in A\} .
\end{aligned}
$$

In the case $B=\left\{x_{0}\right\} \subset X$ we will simply write $x_{0}+A$ instead of $\left\{x_{0}\right\}+A$. Moreover, for $A \subset X$ and a positive integer $n$ we define

$$
\mathcal{S}_{n}(X)=\underbrace{A+\ldots+A}_{n \text { times }} .
$$

Let $D \subset X$ be a non-empty convex set (i.e. $\lambda D+(1-\lambda) D \subset D$ for all $\lambda \in[0,1])$. A function $g: X \rightarrow \mathbb{R}$ is called mid-convex ( $J$-convex) provided $g\left(\frac{x+y}{2}\right) \leq \frac{g(x)+g(y)}{2}$ for $x, y \in D$. Similarly, a function $a: X \rightarrow \mathbb{R}$ is additive if $a(x+y)=a(x)+a(y)$ for $x, y \in X$. The classical results concerning either J-convex functions or additive functions state that boundedness of such functions on sufficiently large sets imply their continuity. In connection with these results R. Ger and M. Kuczma introduced in [7] (for $X=\mathbb{R}^{n}$ ) the following classes of sets:

$$
\begin{aligned}
\mathcal{A}(X)=\{T \subset X: & \text { every mid-convex function } f: D \rightarrow \mathbb{R} \text { bounded above } \\
& \text { on } T \text { is continuous on } D, \text { where } T \subset D \subset X \\
& \text { and } D \text { is nonempty and open }\}, \\
\mathcal{B}(X)=\{T \subset X: & \text { every additive function } a: X \rightarrow \mathbb{R} \text { bounded above } \\
& \text { on } T \text { is continuous }\} .
\end{aligned}
$$

It is known that every additive function is mid-convex so we always have $\mathcal{A}(X) \subset$ $\mathcal{B}(X)$. The equality $\mathcal{A}(X)=\mathcal{B}(X)$ holds for $X$ being a real Baire topological vector space (see [6]), in particular for $X=\mathbb{R}^{n}$ (see [9]).

2010 Mathematics Subject Classification. 39B52, 39B62.

Key words and phrases. Steinhaus-type theorem, Additive function, mid-convex function convex body, point of flatness. 
The question which sets belong to either $\mathcal{A}(X)$ or $\mathcal{B}(X)$ has been a subject of many papers. From the classical results for mid-convex functions (see BersteinDoetsch theorem [3] and its generalization [15]) we get $T \in \mathcal{A}(X)$ provided int $T \neq \emptyset$ in a real topological vector space. This jointly with the property (see [13])

if $\mathcal{S}_{n}(T) \in \mathcal{A}(X)\left(\mathcal{S}_{n} \in \mathcal{B}(X)\right)$ for some $n \geq 2$ then also $T \in \mathcal{A}(X)(T \in \mathcal{B}(X))$, implies

$$
T \in \mathcal{A}(X)(T \in \mathcal{B}(X)) \text { provided } \operatorname{int} \mathcal{S}_{n}(T) \neq \emptyset \text { for some } n \geq 2 .
$$

This nice property leads directly to the Steinhaus-type theorems (theorems of Steinhaus, Piccard and their generalizations) which imply that if $A, B \subset X$ are not small in some sense then int $(A+B) \neq \emptyset$. Sets of positive Lebesgue measure, or sets of the second category with the Baire property are in some sense big ones. However there are known "thin sets" $T$ for which the set $T+T$ has a nonempty interior (and these sets belong to $\mathcal{A}(X)$ and $\mathcal{B}(X))$.

It is known that $C+C=[0,2]$ for the Cantor ternary set, so $C \in \mathcal{A}(\mathbb{R})$. M. Kuczma proved in [12 that the graph of a continuous non-affine function defined on an interval belongs to $\mathcal{A}\left(\mathbb{R}^{2}\right)$. This results has been next generalized for higher dimensions by R. Ger 4 by proving that the regular $(n-1)$-dimensional hypersurface which is not contained in an $(n-1)$-dimensional affine hyperplane belongs to $\mathcal{A}\left(\mathbb{R}^{n}\right)$. In [10] the regularity assumption was weakened and it was proved that the graph of a continuous non-affine function defined on a non-empty open subset of $\mathbb{R}^{n-1}$ is in $\mathcal{A}\left(\mathbb{R}^{n}\right)$. The last results has been generalized to the product space $X \times \mathbb{R}$ for a real continuous function defined on an open subset of a real normed space $X$. R. Ger and M. Sablik shown in 8 , that $S(e, \varepsilon) \in \mathcal{A}(X)$ for arbitrary neighbourhood $S(e, \varepsilon) \subset S_{1}$ of an extremal point $e$ of the unit sphere $S_{1}$ in a real normed space $X$.

P. Volkmann and W. Walter have studied in [16] an equivalent condition for an additive function to be continuous. They have proved that an additive operator $F: X \rightarrow Y$ mapping a real normed space $X$ into a real normed space $Y$ is continuous if and only if $F$ is bounded on a nonempty and (relatively) open subset $A$ of a unit sphere $S_{1}$ in $X$ which is not included in a union of two parallel hyperplanes in $X$. Clearly that result can be simply proved by showing that for such the set $A$ we have $\operatorname{int}(A+A) \neq \emptyset$.

Since the mentioned above conditions lead to sets which algebraic sum of some number copies have interior points we introduce the following notions (cf. also [2]).

Definition 1. Let $X$ be a real topological vector space and fix a positive integer $n \geq 2$. We say that a set $A \subset X$ has $n$-Steinhaus-type property $\left(A \in \mathcal{S P}_{n}(X)\right)$ whenever

$$
\operatorname{int}_{X}\left(\mathcal{S}_{n}(A)\right) \neq \emptyset .
$$

We say that a set $A \subset X$ has strong $n$-Steinhaus-type property $\left(A \in \mathcal{S S P}_{n}(X)\right)$ provided

$$
\operatorname{int}_{X}\left(\mathcal{S}_{n-1}(A)\right)=\emptyset \text { and } \operatorname{int}_{X}\left(\mathcal{S}_{n}(A)\right) \neq \emptyset .
$$

Our aim is to study here "small" sets in a real normed space which have 2Steinhaus-type property. We prove necessary and sufficient condition for subsets of boundary of a convex body to have 2-Steinhaus-type property. Finally we discuss some examples and we pose some problems concerning strong $n$-Steinhaus-type property. 
From now on $(X,\|\cdot\|)$ will be a real normed space and let $\left(X^{*},\|\cdot\|^{*}\right)$ be its dual space. For $v \in X$ let $T_{v}: X \rightarrow X$ be a translation $T_{v}(x)=x+v$ for $x \in X$. Denote by $\bar{B}$ and $B$ the closed unit ball and the open unit ball and in $X$, respectively. Let $S$ and $S^{*}$ be unit spheres in $X$ and in $X^{*}$, respectively.

For arbitrary $x, y \in X$ by $[x, y] \subset X$ we denote the line segment joining points $x$ and $y$, i.e. the set $[x, y]=\{(1-t) x+t y: t \in[0,1]\}$. By a path in $X$ we mean every continuous function $\gamma:[0,1] \rightarrow X$. We will identify a path $\gamma:[0,1] \rightarrow X$ with its image $\Gamma:=\gamma([0,1])=\{\gamma(t) \in X: t \in[0,1]\}$. By a hyperplane in $X$ we mean a set

$$
H_{x_{0}^{*}, c}=\left\{x \in X: x_{0}^{*}(x)=c\right\},
$$

with fixed $x_{0}^{*} \in S^{*}$ and $c \in \mathbb{R}$. Moreover, by

$$
H_{x_{0}^{*}, c}^{-}=\left\{x \in X: x_{0}^{*}(x)<c\right\} \text { and } H_{x_{0}^{*}, c}^{+}=\left\{x \in X: x_{0}^{*}(x)>c\right\},
$$

we denote open halfspaces determined by a hyperplane $H_{x_{0}^{*}, c}$. A path $\gamma:[0,1] \rightarrow X$ is called plane path provided $\gamma([0,1]) \subset H_{x_{0}^{*}, c}$ for some $x_{0}^{*} \in S^{*}$ and $c \in \mathbb{R}$, that is if $x_{0}^{*}(\gamma(t))=c$ for $t \in[0,1]$. In this case we call $\gamma$ the $x_{0}^{*}$-plane path.

A nonempty set $A \subset X$ is called convex provided $[x, y] \subset A$ for every $x, y \in A$. A closed and convex set with interior points we will call a convex body. By the well known Hahn-Banach theorem (see also [14]) we get that for arbitrary convex body $A \subset X$ and for every $x_{0} \in \partial A$ there exist $x_{0}^{*} \in S^{*}$ and $c \in \mathbb{R}$ such that $x_{0}^{*}\left(x_{0}\right)=c$ and $x_{0}^{*}(x) \leq c$ for every $x \in A$. This means that through every boundary point of a convex body there passes a plane supporting the body. In particular, for $\bar{B} \subset X$ and for every $x_{0} \in S=\partial \bar{B}$ there exists $x_{0}^{*} \in S^{*}$ such that $x_{0}^{*}\left(x_{0}\right)=\left\|x_{0}\right\|=1$ and $x_{0}^{*}(x) \leq 1$ for every $x \in \bar{B}$. Clearly the existing $x_{0}^{*}$ need not be unique, so by $\partial A\left(x_{0}\right)\left(S\left(x_{0}\right)\right.$, respectively) we denote the set of all $x_{0}^{*} \in S^{*}$ satisfying $x_{0}^{*}\left(x_{0}\right)=c$ and $x_{0}^{*}(x) \leq c$ for $x \in A\left(x_{0}^{*}\left(x_{0}\right)=1\right.$ and $x_{0}^{*}(x) \leq 1$ for $x \in \bar{B}$, respectively). For a conved body $A \subset X$ a point $x_{0} \in \partial A$ is called a point of flattening of $\partial A$, if there exists $\varepsilon>0$ such that $\partial A \cap\left(x_{0}+\varepsilon B\right) \subset H_{x_{0}^{*}, c}$ for some $x_{0}^{*} \in \partial A\left(x_{0}\right)$ and $c \in \mathbb{R}$, i.e. if $x_{0}^{*}(x)=c$ for $x \in \partial A \cap\left(x_{0}+\varepsilon B\right)$. Note that if $x_{0} \in \partial A$ is a flattening point of $\partial A$, then $\operatorname{card} \partial A\left(x_{0}\right)=1$.

The convex hull, closure, interior, boundary and of a set $A \subset X$ will be indicated by $\operatorname{conv} A, \operatorname{cl} A$ and $\operatorname{int} A$. The set $A_{\varepsilon}=A+\varepsilon B=\{a+\varepsilon b: a \in A, b \in B\}$ will be an $\varepsilon$-neighrbourhood of $A$.

\section{Stainhaus-Type Results}

We prove here our results for the unit sphere. We begin with the following lemma.

Proposition 1. Let us fix $\varepsilon \in(0,1)$ and $x_{0} \in S$. Let $\gamma:[0,1] \rightarrow X$ be a path, which is not $x_{0}^{*}$-plane for some $x_{0}^{*} \in S\left(x_{0}\right)$. Then there are $\alpha, \eta>0$ and $t_{0} \in[0,1]$ such that

$$
\left(\Gamma-\gamma\left(t_{0}\right)+(1-\alpha) x_{0}+z\right) \cap\left(S \cap\left(x_{0}+\varepsilon B\right)\right) \neq \emptyset \quad \text { for all } z \in \eta B .
$$

Proof. Let us fix $\varepsilon \in(0,1), x_{0} \in S$ and consider a neighbourhood $S \cap\left(x_{0}+\varepsilon \bar{B}\right)$ in a relative topology on $S$. If $\gamma$ be a non $x_{0}^{*}$-plane path in $X$ for some $x_{0}^{*} \in S\left(x_{0}\right)$ then for

$$
m:=\inf \left\{\left(x_{0}^{*} \circ \gamma\right)(t): t \in[0,1]\right\} \text { and } M:=\sup \left\{\left(x_{0}^{*} \circ \gamma\right)(t): t \in[0,1]\right\}
$$

we have $M-m>0$. Moreover, $x_{0}^{*} \circ \gamma$ is a continuous function on a compact interval $[0,1]$, so there are $t_{1}, t_{2} \in[0,1]$ with $\left(x_{0}^{*} \circ \gamma\right)\left(t_{1}\right)=m$ and $\left(x_{0}^{*} \circ \gamma\right)\left(t_{2}\right)=M$. 
Without loss of generality we may assume that $t_{0}=0$ and $t_{2}=1$ (if $t_{1}>t_{2}$ we should change the orientation of our path i.e. we shall take the path $\gamma_{1}:[0,1] \rightarrow X$, $\gamma_{1}(t)=\gamma(1-t)$; we have to take next a restriction $\left.\gamma\right|_{\left[t_{1}, t_{2}\right]}$ or $\left.\left.\gamma_{1}\right|_{\left[t_{1}, t_{2}\right]}\right)$. Finally $\gamma$ is uniformly continuous as a continuous function on a compact interval $[0,1]$. Therefore we find $\delta>0$ such that

$$
\|\gamma(s)-\gamma(t)\|<\frac{\varepsilon}{2} \quad \text { for all } s, t \in[0,1] \text { such that }|s-t|<\delta .
$$

Let $n \in \mathbb{N}$ be such that $n \delta \geq 1$. We show that there exist $t_{0}, t_{1} \in[0,1]$ such that $t_{0}<t_{1}<t_{0}+\delta$ and

$$
\begin{array}{ll}
\gamma(t) \in \gamma\left(t_{0}\right)+\frac{\varepsilon}{2} B & \text { for } t \in\left[t_{0}, t_{1}\right], \\
\left(x_{0}^{*} \circ \gamma\right)\left(t_{1}\right)-\left(x_{0}^{*} \circ \gamma\right)\left(t_{0}\right) \geq \frac{M-m}{n} . &
\end{array}
$$

Indeed, for arbitrary $t_{0}, t_{1} \in[0,1]$ with $t_{0}<t_{1}$ and $t_{1}-t_{0}<\delta$, from (2) we get

$$
\left\|\gamma(t)-\gamma\left(t_{0}\right)\right\|<\frac{\varepsilon}{2} \quad \text { for } t \in\left[t_{0}, t_{1}\right] .
$$

This implies (3). In order to prove (4) let us suppose that contrary to (4) we have

$$
\left(x_{0}^{*} \circ \gamma\right)\left(t_{1}\right)-\left(x_{0}^{*} \circ \gamma\right)\left(t_{0}\right)<\frac{M-m}{n}
$$

for all $t_{0}, t_{1} \in[0,1]$ such that $t_{0}<t_{1}$ and $t_{1}-t_{0}<\delta$. Since $n \delta \geq 1$ we can find $0=s_{0}<s_{1}<\ldots<s_{n}=1$ such that $s_{k}-s_{k-1}<\delta$ for $k \in\{1, \ldots, n\}$. Then by (5) we obtain

$$
\left(x_{0}^{*} \circ \gamma\right)\left(s_{k}\right)-\left(x_{0}^{*} \circ \gamma\right)\left(s_{k-1}\right)<\frac{M-m}{n} \quad \text { for } k \in\{1, \ldots, n\} .
$$

Thus

$$
\begin{aligned}
M-m & =\left(x_{0}^{*} \circ \gamma\right)(1)-\left(x_{0}^{*} \circ \gamma\right)(0) \\
& =\sum_{k=1}^{n}\left(\left(x_{0}^{*} \circ \gamma\right)\left(s_{k}\right)-\left(x_{0}^{*} \circ \gamma\right)\left(s_{k-1}\right)\right) \\
& <\sum_{k=1}^{n} \frac{M-m}{n}=n \cdot \frac{M-m}{n}=M-n .
\end{aligned}
$$

This contradiction implies that (4) holds true for some $t_{0}, t_{1} \in[0,1]$ such that $t_{0}<t_{1}<t_{0}+\delta$.

Put $\alpha:=\min \left(\frac{M-m}{2 n}, \frac{\varepsilon}{4}\right)$ and define $\widetilde{\gamma}:[0,1] \rightarrow X$,

$$
\widetilde{\gamma}(t)=\left(T_{-\gamma\left(t_{0}\right)+(1-\alpha) x_{0}} \circ \gamma\right)(t)=\gamma(t)-\gamma\left(t_{0}\right)+(1-\alpha) x_{0} \quad \text { for } t \in[0,1] .
$$

Hence $\widetilde{\Gamma}=\Gamma-\gamma\left(t_{0}\right)+(1-\alpha) x_{0}$. Then $\widetilde{\gamma}\left(t_{0}\right)=(1-\alpha) x_{0}$ and $\left\|\alpha x_{0}\right\| \leq \frac{\varepsilon}{4}$, so $\widetilde{\gamma}\left(t_{0}\right)=(1-\alpha) x_{0} \in x_{0}+\frac{\varepsilon}{4} \bar{B}$ and $\left\|\widetilde{\gamma}\left(t_{0}\right)\right\|=\left\|(1-\alpha) x_{0}\right\|<1$. Moreover, $\left(x_{0}^{*} \circ \widetilde{\gamma}\right)\left(t_{0}\right)=(1-\alpha) x_{0}^{*}\left(x_{0}\right)=1-\alpha<1$. Furthermore, from (3) for every $t \in\left[t_{0}, t_{1}\right]$ we get

$$
\widetilde{\gamma}(t)=\gamma(t)-\gamma\left(t_{0}\right)+(1-\alpha) x_{0} \in \gamma\left(t_{0}\right)-\gamma\left(t_{0}\right)+\frac{\varepsilon}{2} B+(1-\alpha) x_{0} \subset x_{0}+\frac{3 \varepsilon}{4} B,
$$


which means that $\widetilde{\gamma}\left(\left[t_{0}, t_{1}\right]\right) \subset x_{0}+\frac{3 \varepsilon}{4} B$. Finally, by (44) we have

$$
\begin{aligned}
\left(x_{0}^{*} \circ \widetilde{\gamma}\right)\left(t_{1}\right) & =x_{0}^{*}\left(\gamma\left(t_{1}\right)-\gamma\left(t_{0}\right)+(1-\alpha) x_{0}\right) \\
& =\left(x_{0}^{*} \circ \gamma\right)\left(t_{1}\right)-\left(x_{0}^{*} \circ \gamma\right)\left(t_{0}\right)+(1-\alpha) x_{0}^{*}\left(x_{0}\right) \\
& \geq \frac{M-m}{n}+\left(1-\frac{M-m}{2 n}\right)=1+\frac{M-m}{2 n}>1,
\end{aligned}
$$

so $\left\|\widetilde{\gamma}\left(t_{1}\right)\right\|>1$.

We have thus proved that $\widetilde{\gamma}\left(t_{0}\right)$ is an interior point of the set $\left(x_{0}+\frac{\varepsilon}{2} B\right) \cap B$, $\widetilde{\gamma}\left(\left[t_{0}, t_{1}\right]\right) \subset x_{0}+\frac{3 \varepsilon}{4} B$ and $\widetilde{\gamma}\left(t_{1}\right)$ is an interior point of the set $\left(x_{0}+\frac{3 \varepsilon}{4} B\right) \cap H_{x_{0}^{*}, 1}^{+}$. Fix $\eta<\alpha$ such that $\widetilde{\gamma}\left(t_{0}\right)+\eta B \subset\left(x_{0}+\frac{\varepsilon}{2} B\right) \cap B$ and $\widetilde{\gamma}\left(t_{1}\right)+\eta B \subset\left(x_{0}+\frac{3 \varepsilon}{4} B\right) \cap H_{x_{0}^{*}, 1}^{+}$.

For arbitrary $z \in \eta B$ we define $\widehat{\gamma}_{z}:\left[t_{0}, t_{1}\right] \rightarrow X$ by

$$
\widehat{\gamma}_{z}(t)=\left(T_{z} \circ \widetilde{\gamma}\right)(z)=\gamma(t)-\gamma\left(t_{0}\right)+(1-\alpha) x_{0}+z \quad \text { for } t \in\left[t_{0}, t_{1}\right],
$$

and let $\widehat{\Gamma}_{z}:=\left\{\widehat{\gamma}_{z}(t): t \in\left[t_{0}, t_{1}\right]\right\}$. For every $t \in\left[t_{0}, t_{1}\right]$ we have

$$
\widehat{\gamma}_{z}(t)=\left(T_{z} \circ \widetilde{\gamma}\right)(t)=\widetilde{\gamma}(t)+z \in x_{0}+\frac{3 \varepsilon}{4} B+\eta B \subset x_{0}+\frac{3 \varepsilon}{4} B+\frac{\varepsilon}{4} B=x_{0}+\varepsilon B,
$$

hence $\widetilde{\Gamma}_{z} \subset x_{0}+\varepsilon B$ for every $z \in \eta B$. Moreover,

$$
\widehat{\gamma}_{z}\left(t_{0}\right)=\widetilde{\gamma}\left(t_{0}\right)+z \in \widetilde{\gamma}\left(t_{0}\right)+\eta B \subset\left(x_{0}+\frac{\varepsilon}{2} B\right) \cap B \subset\left(x_{0}+\varepsilon B\right) \cap B .
$$

Finally, $\left\|x_{0}^{*}\right\|^{*}=1$ and $\eta<\alpha \leq \frac{M-m}{2 n}$, so $\|z\|<\frac{M-m}{2 n}$,

$$
\begin{aligned}
\left(x_{0}^{*} \circ \widehat{\gamma}_{z}\right)\left(t_{1}\right) & =x_{0}^{*}\left(\gamma\left(t_{1}\right)-\gamma\left(t_{0}\right)+(1-\alpha) x_{0}+z\right) \\
& =x_{0}^{*}\left(\gamma\left(t_{1}\right)\right)-x_{0}^{*}\left(\gamma\left(t_{0}\right)\right)+(1-\alpha) x_{0}^{*}\left(x_{0}\right)-x_{0}^{*}(-z) \\
& \geq \frac{M-m}{n}+\left(1-\frac{M-m}{2 n}\right)-\left\|x_{0}^{*}\right\| \cdot\|z\| \\
& >1+\frac{M-m}{2 n}-\frac{M-m}{2 n}=1,
\end{aligned}
$$

and

$$
\widehat{\gamma}_{z}\left(t_{1}\right)=\widetilde{\gamma}\left(t_{1}\right)+z \in \widetilde{\gamma}\left(t_{1}\right)+\eta B \subset\left(x_{0}+\frac{3 \varepsilon}{4} B\right) \cap H_{x_{0}^{*}, 1}^{+} \subset\left(x_{0}+\varepsilon B\right) \cap H_{x_{0}^{*}, 1}^{+} .
$$

Let $\left|\widehat{\gamma}_{z}\right|:\left[t_{0}, t_{1}\right] \rightarrow \mathbb{R}$ be defined by

$$
\left|\widehat{\gamma}_{z}\right|(t)=\left\|\widehat{\gamma}_{z}(t)\right\| \quad \text { for } t \in\left[t_{0}, t_{1}\right]
$$

Since $\widehat{\gamma}_{z}\left(t_{0}\right) \in B$, so $\left|\widehat{\gamma}_{z}\right|\left(t_{0}\right)<1$. Similarly, $\widehat{\gamma}_{z}\left(t_{1}\right) \in H_{x_{0}^{*}, 1}^{+}$, so $\widehat{\gamma}_{z}\left(t_{1}\right) \in X \backslash \bar{B}$, hence $\left|\widehat{\gamma}_{z}\left(t_{1}\right)\right|\left(t_{1}\right)>1$. By continuity of $\left|\widehat{\gamma}_{z}\right|$ there exists $t_{z} \in\left[t_{0}, t_{1}\right]$ such that $\left|\widehat{\gamma}_{z}\right|\left(t_{z}\right)=\left\|\widehat{\gamma}_{z}\left(t_{z}\right)\right\|=1$ and, moreover, $\widehat{\gamma}_{z}\left(t_{z}\right) \in\left(x_{0}+\varepsilon B\right)$. Thus

$$
\widehat{\Gamma}_{z} \cap\left(S \cap\left(x_{0}+\varepsilon B\right)\right) \neq \emptyset \quad \text { for each } z \in \eta B .
$$

This finishes the proof.

From proposition we derive the following result.

Theorem 1. Let us fix $\varepsilon \in(0,1), x_{0} \in S$ and let $\gamma:[0,1] \rightarrow X$ be a path, which is not $x_{0}^{*}$-plane for some $x_{0}^{*} \in S\left(x_{0}\right)$. Then $\operatorname{int}\left(\left(S \cap\left(x_{0}+\varepsilon B\right)\right) \pm \Gamma\right) \neq \emptyset$. 
Proof. Fix $\varepsilon \in(0,1), x_{0} \in S$ and assume that $\gamma:[0,1] \rightarrow X$ is a path, which is not $x_{0}^{*}$-plane for some $x_{0}^{*} \in S\left(x_{0}\right)$. By Proposition 1 there exist $\alpha, \eta>0$ and $t_{0} \in[0,1]$ such that (11) holds. Hence for arbitrary $z \in \eta B$ we find $a \in S \cap\left(x_{0}+\varepsilon B\right)$ such that $a \in \Gamma-\gamma\left(t_{0}\right)+(1-\alpha) x_{0}+z$. This implies that $a=b-\gamma\left(t_{0}\right)+(1-\alpha) x_{0}+z$ for some $b \in \Gamma$. Thus

$-\gamma\left(t_{0}\right)+(1-\alpha) x_{0}+\eta B \ni-\gamma\left(t_{0}\right)+(1-\alpha) x_{0}+z=a-b \in\left(S \cap\left(x_{0}+\varepsilon B\right)\right)-\Gamma$, so $\operatorname{int}\left(\left(S \cap\left(x_{0}+\varepsilon B\right)\right)-\Gamma\right) \neq \emptyset$.

To prove int $\left(\left(S \cap\left(x_{0}+\varepsilon B\right)\right)+\Gamma\right) \neq \emptyset$ it is enough to apply Proposition 1 for the path $-\Gamma$. Indeed, if $\Gamma$ is not $x_{0}^{*}$-plane, then also $-\Gamma$ is not $x_{0}^{*}$-plane. Then for arbitrary $z \in \eta B$ we find $a^{\prime} \in S \cap\left(x_{0}+\varepsilon B\right)$ such that $a^{\prime} \in-\Gamma+\gamma\left(t_{0}\right)+(1-\alpha) x_{0}+z$. Thus $a^{\prime}=-b^{\prime}+\gamma\left(t_{0}\right)+(1-\alpha) x_{0}+z$ for some $b^{\prime} \in \Gamma$ and

$$
\begin{gathered}
\gamma\left(t_{0}\right)+(1-\alpha) x_{0}+\eta B \ni \gamma\left(t_{0}\right)+(1-\alpha) x_{0}+z=a+b \in\left(S \cap\left(x_{0}+\varepsilon B\right)\right)+\Gamma, \\
\text { so } \gamma\left(t_{0}\right)+(1-\alpha) x_{0}+\eta B \subset\left(S \cap\left(x_{0}+\varepsilon B\right)\right)+\Gamma \text { and } \operatorname{int}\left(\left(S \cap\left(x_{0}+\varepsilon B\right)\right)+\Gamma\right) \neq \emptyset .
\end{gathered}
$$

From Theorem! 1] we obtain the following crucial corollary.

Corollary 1. Fix $x_{0} \in S$. Then $x_{0}$ is not a flattening point of $S$ if and only if

$$
\operatorname{int}\left(\left(S \cap\left(x_{0}+\varepsilon B\right)\right)+\left(S \cap\left(x_{0}+\varepsilon B\right)\right)\right) \neq \emptyset \quad \text { for arbitrary small } \varepsilon>0 .
$$

Proof. Let us fix $x_{0} \in S$. Assume first that $x_{0}$ is a flattening point of $S$. Then there exist $\varepsilon>0$ and $x_{0}^{*} \in S\left(x_{0}\right)$ such that $x_{0}^{*}(x)=1$ for all $x \in S \cap\left(x_{0}+\varepsilon B\right)$. Thus

$$
x_{0}^{*}\left(x_{1}+x_{2}\right)=x_{0}^{*}\left(x_{1}\right)+x_{0}^{*}\left(x_{2}\right)=2 \quad \text { for all } x_{1}, x_{2} \in S \cap\left(x_{0}+\varepsilon B\right),
$$

so int $\left(\left(S \cap\left(x_{0}+\varepsilon B\right)\right)+\left(S \cap\left(x_{0}+\varepsilon B\right)\right)\right)=\emptyset$.

Let us assume now that $x_{0}$ is not a flattening point of $S$. Then for all $x_{0}^{*} \in S\left(x_{0}\right)$ and arbitrary small $\varepsilon>0$ there exists $z_{0} \in S \cap\left(x_{0}+\varepsilon B\right)$ such that $x_{0}^{*}\left(z_{0}\right)<1$. Let us fix $x_{0}^{*} \in S\left(x_{0}\right), \varepsilon \in(0,1)$ and $z_{0} \in S \cap\left(x_{0}+\varepsilon B\right)$ with $x_{0}^{*}\left(z_{0}\right)<1$. We consider a path $\gamma:[0,1] \rightarrow S$,

$$
\gamma(t)=\left\|(1-t) x_{0}+t z_{0}\right\| \quad \text { for } t \in[0,1] .
$$

Then $\gamma$ is not $x_{0}^{*}$-plane, and by Theorem 1 we get

$$
\operatorname{int}\left(\left(S \cap\left(x_{0}+\varepsilon B\right)\right)+\left(S \cap\left(x_{0}+\varepsilon B\right)\right)\right) \supset \operatorname{int}\left(\left(S \cap\left(x_{0}+\varepsilon B\right)\right)+\Gamma\right) \neq \emptyset .
$$

This finishes the proof.

From Corollary 1 one can easily derive the result proved in [8]. Moreover, it is clear, that the result proved above is invariant with respect to translations $X \ni$ $x \mapsto x+v \in X$ and uniform scalings $X \ni x \mapsto \lambda x \in X$ with fixed $v \in X$ and $\lambda \neq 0$. Thus we have the following result.

Corollary 2. For every $\delta>0$ and for all $x \in X, r>0$ we have

$$
x+r \mathcal{S}_{1}\left(x_{0}, x_{0}^{*}, \delta\right) \in \mathcal{A}(X) \cap \mathcal{B}(X) .
$$

Using Theorem 1 and Corollary 11 we get also another proof or theorem by P. Volkmann and W. Walter [16].

Corollary 3. Let $X$ be a real normed space of dimension at least 2. If $A \subset S_{1}$ is a nonempty and (relatively) open subset of a unit sphere in $X$ which is not included in a union of two parallel hyperplanes in $X$, then $\operatorname{int}(A+A) \neq \emptyset$ and hence $A \in \mathcal{A}(X) \cap \mathcal{B}(X)$. 
Proof. If $A \subset S_{1}$ is a nonempty and (relatively) open subset of a unit sphere in $X$ which is not included in a union of two parallel hyperplanes then we have the following two possibilities:

(a) $A$ contains a point and its relatively open in $S_{1}$ neighbourhood which not $x^{*}$-flat for every $x^{*} \in S_{1}^{*}$,

(b) A contains two relatively open components contained in two different nonparalel hyperplanes.

In the first case we use Corollary 1 to obtain our statement. In the second case we take any relatively open $x^{*}$-plane subset of the first component and arbitrary line segment contained in the second one which is clearly not $x^{*}$-plane. Then Theorem 1 finishes the proof.

We are in position to discuss now the general case of a convex body in $X$. We prove

Theorem 2. Let $K \subset X$ be a convex body and fix $x_{0} \in \partial K$. Then $x_{0}$ is not a flattening point of $\partial K$ if and only if

$$
\operatorname{int}\left(\left(\partial K \cap\left(x_{0}+\varepsilon B\right)\right)+\left(\partial K \cap\left(x_{0}+\varepsilon B\right)\right)\right) \neq \emptyset \quad \text { for arbitrary small } \varepsilon>0 .
$$

Proof. Let $\|\cdot\|$ be a norm in $X$. Fix $x_{0} \in \partial K$ and let $z_{0} \in \operatorname{int} K$. Since the property which we want to prove is invariant with respect to translations without loss of generality we can assume that $z_{0}=0$. Let $\delta:=\left\|x_{0}\right\|$ and consider $A:=$ $\partial K \cap\left(x_{0}+\frac{\delta}{2} B\right)$. Then the set $V:=\operatorname{cl} \operatorname{conv}\left(A \cup \frac{\delta}{4} B \cup(-A)\right)$ is closed, convex, bounded, symmetric with respect to 0 and contains 0 in its interior. Thus the Minkowski's functional $\mu_{V}$ properly defines a norm $\|\cdot\|_{V}$ equivalent to $\|\cdot\|$ in $X$. Obviously dual spaces for $(X,\|\cdot\|)$ and $\left(X,\|\cdot\|_{V}\right)$ coincides. Let $S_{V}$ and $B_{V}$ be unit sphere and unit ball in the norm $\|\cdot\|_{V}$, respectively. Then for sufficiently small $\varepsilon>0$ we have

$$
S_{V} \cap\left(x_{0}+\varepsilon B_{V}\right) \subset \partial K \cap\left(x_{0}+\frac{\delta}{2} B\right) .
$$

Then from Corollary 1 we get our statement.

\section{EXAMPLES AND PROBLEMS}

We begin with the following simple and obvious example.

Example 1. For every $n \in \mathbb{N}$ with $n \geq 2$ in the space $\mathbb{R}^{n}$ there exists a set $A \in \mathcal{S} \mathcal{S P}{ }_{n}\left(\mathbb{R}^{n}\right)$. If $e_{i}$ for $i \in\{0,1, \ldots, n\}$ is a canonical affine basis of $\mathbb{R}^{n}$, then the polyline joining points $e_{0}, e_{1}, \ldots, e_{n}$ has the desired property.

Based on the above example we can ask the following question.

Problem 1. Fix $n \in \mathbb{N}$ with $n \geq 2$. Let $\gamma:[0,1] \rightarrow \mathbb{R}^{n}$ define a continuous curve $\Gamma$ which goes through $(n+1)$ affinely independent points in $\mathbb{R}^{n}$. Is it true that $\Gamma \in \mathcal{S P}_{n}\left(\mathbb{R}^{n}\right)$ ?

Using Theorem 1.3 (a) and (e) from [1] we get the next example.

Example 2. Let $C_{\lambda}$ be a Cantor-type set with selfsimilarity ratio $\lambda$. For $k \in \mathbb{N}$ with $k \geq 2$ and for $\lambda \in\left[\frac{1}{k+1}, \frac{1}{k}\right)$ we have $C_{\lambda} \in \mathcal{S S P}_{k}(\mathbb{R})$ that is

$$
\operatorname{int}\left(\mathcal{S}_{k-1}\left(C_{\lambda}\right)\right)=\emptyset \quad \text { and } \quad \mathcal{S}_{k}\left(C_{\lambda}\right)=[0, k] .
$$

We can generalize this example to higher dimensions. 
Example 3. Let $C_{\lambda}$ be a Cantor-type set with selfsimilarity ratio $\lambda$. For $k, n \in \mathbb{N}$ with $k, n \geq 2$ and for $\lambda \in\left[\frac{1}{n+1}, \frac{1}{n}\right)$ we have $C_{\lambda} \times[0,1]^{n-1} \in \mathcal{S S P}_{k}\left(\mathbb{R}^{n}\right)$ that is

$$
\operatorname{int}\left(\mathcal{S}_{k-1}\left(C_{\lambda}\right)\right)=\emptyset \quad \text { and } \quad \mathcal{S}_{k}\left(C_{\lambda}\right)=[0, k]^{n} \text {. }
$$

\section{REFERENCES}

[1] T. Banakh, A. Bartoszewicz, M. Filipczak, E. Szymonik, Topological and measure properties of some self-similar sets, Topol. Methods Nonlinear Anal. 46 (2015), 1013-1028.

[2] T. Banakh, L. Karchevska, A. Ravsky, The closed Steinhaus properties of $\sigma$-ideals on topological groups, https://arxiv.org/abs/1509.09073v1

[3] F. Bernstein, G. Doetsch, Zur Theorie der konvexen Funktionen, Math. Ann. 76 (1915), 514-526.

[4] Ger R., Note on convex functions bounded on regular hypersurfaces, Demonstratio Math. vol. 6 (1973), 97-103.

[5] Ger R., Thin sets and convex functions, Bull. Acad. Polon. Sci. Sér. Sci. Math. Astronom. Phys. 21 (1973), 413-416.

[6] R. Ger, Z. Kominek, Boundedness and continuity of additive and convex functionals, Aequationes Math. 37 (1989), 252-258.

[7] Ger R., Kuczma M., On the boundedness and continuity of convex functions and additive functions, Aequationes Math. 4 (1970), 157-162.

[8] Ger R., SABlik M., Homomorphisms and boundedness in topological groups, Aequat. Math. 80 (2010), 119-130.

[9] M. E. Kuczma, On discontinuous additive functions, Fund. Math. 66 (1969/1970), 383-392.

[10] Jabłoński W., On a class of sets connected with a convex function, Abh. Math. Sem. Univ. Hamburg 69 (1999), 205-210.

[11] JABŁOŃSKI W., Sum of graphs of continuous functions and boundedness of additive operators, Journal of Math. Anal. Appl. 312 (2005), 527-534.

[12] Kuczma M., On some set classes occurring in the theory of convex functions, Annales Soc. Math. Pol., Comment. Math. 17 (1973), 127-135.

[13] Kuczma M., An introduction to the theory of functional equations and inequalities. Cauchy's equation and Jensens inequality. In: Gilànyi, A. (ed.) 2nd edn. Birkhäuser Verlag, Basel 2009.

[14] Mazur S., Über konvexe Mengen in linearen normierte Räumen, Studia Math. 4 (1933), $70-84$.

[15] Ja. Tabor, Jo. Tabor, M. Żołdak, Approximately convex functions on topological vector spaces, Publ. Math. Debrecen 77 (2010), 115-123.

[16] Volkmann P., Walter W., Condition for the continuity of additive operators, Ann. of Diff. Eqs. 3(1) 1987, 63-66.

Department of Mathematical Modeling, Faculty of Mathematics and Applied Physics, Rzeszów University of Technology, Powstańców Warszawy 6, PL-35-959 Rzeszów 